\title{
HEAVY QUARKONIUM*
}

\author{
T. SKWARNICKI \\ Department of Physics, Syracuse University, NY 13244, USA \\ E-mail: tomasz@physics.syr.edu
}

\begin{abstract}
I review heavy quarkonium physics in view of recent experimental results. In particular, I discuss new results on spin singlet states, photon and hadronic transitions, $D$-states and discovery of the yet unexplained narrow $X(3872)$ state.
\end{abstract}

\section{Quarkonia}

Quarkonium is a bound state of a quark and its antiquark. Some properties of light and heavy quarkonia are compared to properties of positronium in Table 1. Unlike positronium, light quarkonia are highly relativistic. They also contain mixtures of quarks of different flavor and fall apart easily into other mesons. Charmonium $(c \bar{c})$ was the first heavy quarkonium discovered and is less relativistic; the number of longlived states below the dissociation energy (i.e. the threshold for decay to $D \bar{D}$ meson pairs) equals the number of long-lived positronium states. Bottomonium $(b \bar{b})$ is even more non-relativistic and has a larger number of long-lived states. The toponium system would have been completely non-relativistic. However, weak decays of the top quark will dominate over the strong binding and long-lived states will not be formed. Therefore, charmonium and bottomonium play a special role in probing strong interactions.

The states below open flavor threshold live long enough for electromagnetic transitions between various excitations to occur. The electromagnetic transitions compete with transitions mediated by the emission of soft gluons. The latter materialize as light hadrons. Eventually the heavy quarks must annihilate into two or three hard gluons. Properties of these bound states and their decays are good testing grounds for QCD in both the non-perturbative and perturbative regimes.

The first heavy quarkonium bound state above the $D \bar{D}$ or $B \bar{B}$ threshold acts as a factory of heavylight mesons. The heavy quarks trapped in these mesons ultimately decay via weak interactions. This is a good place to look for physics beyond the stan-

\footnotetext{
*Invited talk presented at the $21^{\text {st }}$ International Symposium On Lepton And Photon Interactions At High Energies (LP03) 11-16 August 2003, Batavia, Illinois.
}

dard model as discussed by Y. Grossman at this Symposium. ${ }^{1}$ Many measurements of electroweak parameters are obscured by strong interactions. This provides an important motivation for trying to understand details of strong interaction phenomena. P. Lepage pointed out that at least some of the new interactions to be discovered are likely to be strongly coupled, further motivating detailed studies of QCD. ${ }^{2}$

Heavy quarkonia offer two small parameters: velocities $(v)$ of constituent quarks and the strong coupling constant $\left(\alpha_{s}\right)$ in annihilation and production processes. The expansion of the full theory in these parameters allows for effective theories of strong interactions: in the past - purely phenomenological potential models; more recently - NRQCD. ${ }^{3}$ Lattice QCD calculations are also easier for heavy quarkonia than for light hadrons.

\section{Hadroproduction}

Annihilation of $n^{3} S_{1^{--}}(\psi, \Upsilon)$ states to $\mu^{+} \mu^{-}$and to a lesser extent $e^{+} e^{-}$makes it possible for experimentalists to fish out these states from large backgrounds in hadroproduction experiments. This is how these states were (co)discovered! There is also some access to the production of the excited states by addition of a transition photon or $\pi^{+} \pi^{-}$pair. So far, except for the initial discovery, the hadroproduction experiments have not played an important role in spectroscopy or decay studies, but have made interesting production measurements. Heavy quarkonium is also a useful probe for determining the structure of the target (e.g. to probe for gluon content or the presence of quark-gluon plasma).

Non-relativistic QCD (NRQCD) is a favorite theoretical approach used to describe the production data. ${ }^{4}$ Various diagrams are ordered according to powers of $\alpha_{s}$ and $v$. The diagrams can also be 
Table 1. Some properties of various "onia".

\begin{tabular}{|c|c|c|c|c|c|c|c|c|}
\hline \multicolumn{2}{|c|}{ FORCES } & \multirow[t]{2}{*}{ System } & \multicolumn{3}{|c|}{ Ground triplet state $1^{3} S_{1}$} & \multirow[t]{2}{*}{$\left(\frac{v}{c}\right)^{2}$} & \multicolumn{2}{|c|}{$\begin{array}{l}\text { Number of states } \\
\text { below dissociation } \\
\text { energy }\end{array}$} \\
\hline binding & decay & & Name & $\Gamma(\mathrm{MeV})$ & Mass $(\mathrm{GeV})$ & & $n^{3} S_{1}$ & all \\
\hline \multicolumn{9}{|c|}{ POSITRONIUM } \\
\hline EM & $\overline{\mathrm{EM}}$ & $e^{+} e^{-}$ & Ortho- & $5 \times 10^{-15}$ & 0.001 & $\sim 0.0$ & 2 & 8 \\
\hline \multicolumn{9}{|c|}{ QUARKONIUM } \\
\hline \multirow[t]{5}{*}{ Strong } & \multirow[t]{2}{*}{ Strong } & $u \bar{u}, d \bar{d}$ & $\rho$ & 150.00 & 0.8 & $\sim 1.0$ & 0 & $\overline{0}$ \\
\hline & & $s \bar{s}$ & $\phi$ & 4.40 & 1.0 & $\sim 0.8$ & "1" & "2" \\
\hline & \multirow[t]{2}{*}{$\overline{\mathrm{EM}}$} & $c \bar{c}$ & $\bar{\psi}$ & 0.09 & 3.1 & $\sim 0.25$ & 2 & 8 \\
\hline & & $b b$ & $\Upsilon$ & 0.05 & 9.5 & $\sim 0.08$ & 3 & 30 \\
\hline & weak & $t \bar{t}$ & & $(3000.0)$ & $(360)$. & $<0.01$ & 0 & 0 \\
\hline
\end{tabular}

classified into color-singlet processes, in which the heavy $Q \bar{Q}$ pair is produced colorless and therefore can directly form a bound state, and color-octet processes, in which the $Q \bar{Q}$ pair has color which must be emitted away by soft gluon (i.e. long distance) interactions. Initial attempts to describe charmonium production at the Tevatron with only color-singlet processes $(\mathrm{CS} \text { model })^{5}$ failed spectacularly, revealing the importance of color-octet diagrams. However, the color-evaporation model $(\mathrm{CEM}){ }^{6}$ which allows color-octet processes but neglects their ranking in $v$, also fits the data well. All theoretical approaches involve free parameters, which are fixed by fits to the data, diminishing their effective differences.

Polarization of produced heavy quarkonia should lead to a better discrimination between NRQCD and CEM. NRQCD predicts an increase in polarization with higher $p_{t}$, while CEM predicts no polarization at all. The present data do not provide any evidence for increase in polarization with $p_{t}$, however the experimental errors are too large to draw any firm conclusions.

There is a large range of kinematical regimes and differential cross sections for charmonium production studies at HERA. Both H1 and ZEUS contributed a number of papers on this topic to this Symposium. ${ }^{7}$ NRQCD predicts that matrix elements should be universal. However, it is difficult to reconcile all Tevatron and HERA data with a consistent set of NRQCD matrix elements. It is quite possible that the charm quark is just not heavy enough for the NRQCD approximation to work well. Unfortunately, data on bottomonium production are either non-existent or have large experimental errors.

For a more complete review of this topic see e.g.
Krämer. ${ }^{4}$

\section{Clean Production Environments}

Most of what we know about heavy quarkonium states and their decays comes from experiments at clean production environments, which are time reversals of simple decay modes (see Fig. 1).

Vector states $\left(J^{P C}=1^{--}: n^{3} S_{1}, n^{3} D_{1}\right)$ decay to lepton pairs and thus can be directly formed in $e^{+} e^{-}$collisions. Production rates are large compared to the other $e^{+} e^{-}$processes, thus the backgrounds are small and these states can be studied in both inclusive and exclusive decay modes. Dedicated runs are needed for each vector state. The other states can be reached via photon and hadronic transitions, however, their scope is limited by the transition selection rules and branching ratios.

Spin 0 and 2 states with positive C-parity $\left(J^{P C}=0^{-+}, 0^{++}, 2^{++}: n^{1} S_{0}, n^{3} P_{0,2}\right)$ decay to twophotons, thus can be formed in two-photon collisions at the $e^{+} e^{-}$colliders. No dedicated runs are needed since the two-photon flux populates a wide range of possible quarkonia masses. However, the flux drops quickly with the energy and production rates for heavy quarkonia are small compared to the dominant $e^{+} e^{-}$processes. To combat these large backgrounds the quarkonia states must be detected in simple exclusive decay modes. So far this formation method has succeeded only for charmonium states.

States of any quantum numbers can decay to $p \bar{p}$ via two- or three-gluon annihilation, however, such couplings are small. Low energy $\bar{p}$ beams annihilating with proton-jet targets were successfully used to form charmonium states. The beam energy must be 
tuned to form a specific quarkonium state. Since the background cross-sections are very large, the quarkonia must be detected in simple exclusive final states to take advantage of the highly constrained kinematics.

Decays of $B$ mesons also offer a clean production environment for charmonium states. Again states of any quantum number can be formed. Since the production rates are rather small, experimentalists must restrict themselves to specific exclusive final states to take advantage of the $B$ mass constraint (and beam energy constraint if produced at the $\Upsilon(4 S)$ resonance in $e^{+} e^{-}$collisions).

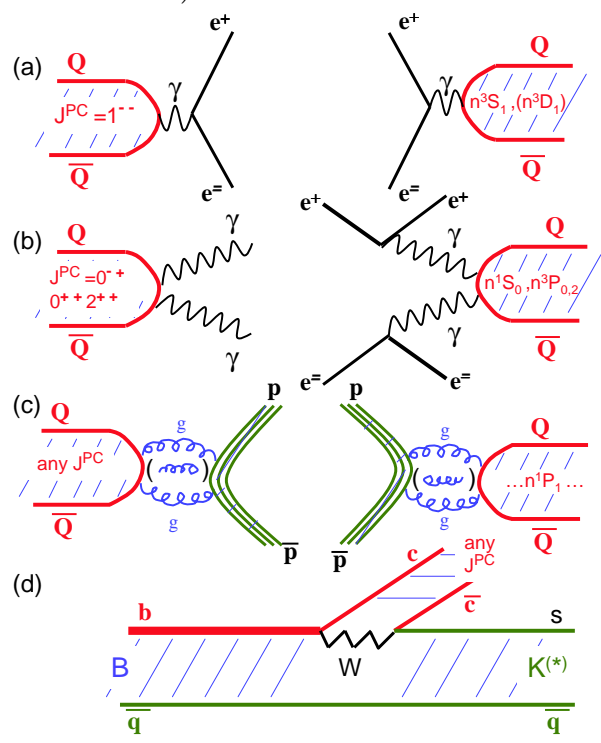

Figure 1. Clean production environments for heavy quarkonia together with corresponding decay modes for most of them.

\section{Results from $\bar{p} p$ Experiments}

A $H_{2}$ gas jet is used as a target for an antiproton beam in the $\bar{p}$ accumulator ring. This technique was pioneered at the CERN-ISR by the R704 experiment (1983-84). ${ }^{8}$ It was later used at Fermilab with the E760 detector (1989-91) ${ }^{9}$ followed by its major upgrade E835 (1996-97, 2000). Since this was a nonmagnetic detector, it was limited to specific final states containing electrons and photons. Charmonium states are observed directly as resonance peaks in the signal event yield measured as function of the center-of-mass energy. Masses and widths of the observed states can be measured with high precision, since the accuracy depends on knowledge of the beam energy rather than resolution of the detector. The Fermilab experiments determined masses and widths of the $\chi_{c J}$ states with unprecedented accuracy. ${ }^{9,10}$ They also studied radiative transitions from these states. ${ }^{11}$ The most recent results include detection of the $\chi_{c 0}$ resonance in decays to $\pi^{0} \pi^{0}$, interfering with continuum production of this final state ${ }^{12}$ and observation of the singlet $\eta_{c}\left(1^{1} S_{0}\right)$ state in annihilation to two photons. ${ }^{13}$ The radial excitation of the latter was not found in scans by E760 and E835. ${ }^{14}$ Only the region around the mass reported by the Crystal Ball experiment $^{15}$ was scanned.

While the singlet $\eta_{c}\left(1^{1} S_{0}\right)$ was observed in many different environments, the only hints for the singlet $h_{c}\left(1^{1} P_{1}\right)$ state come from the $\bar{p} p$ experiments. R704 provided some inconclusive evidence for this state in $1984 .^{16}$ Better evidence for this state, with the mass close to the center-of-gravity mass of the $\chi_{c}\left(1^{3} P_{J}\right)$ states, was reported by E760 $0^{17}$ via the decay chain: $h_{c} \rightarrow \pi^{0} J / \psi, J / \psi \rightarrow e^{+} e^{-}$. E835 took more scans in this mass range, with larger statistics and an improved detector. Recently, rumors of absence of the $h_{c}$ state in the E835 data was reported in print by several non-E835 authors. ${ }^{18}$ However, the official word from the E835 collaboration $^{19}$ is that they are analyzing all available decay channels and are not ready to make any definite statements yet.

Average: $(2979.9 \pm 1.0) \mathrm{MeV}$ $\mathrm{CL}=0.5 \%$ Scale Factor $=1.5$

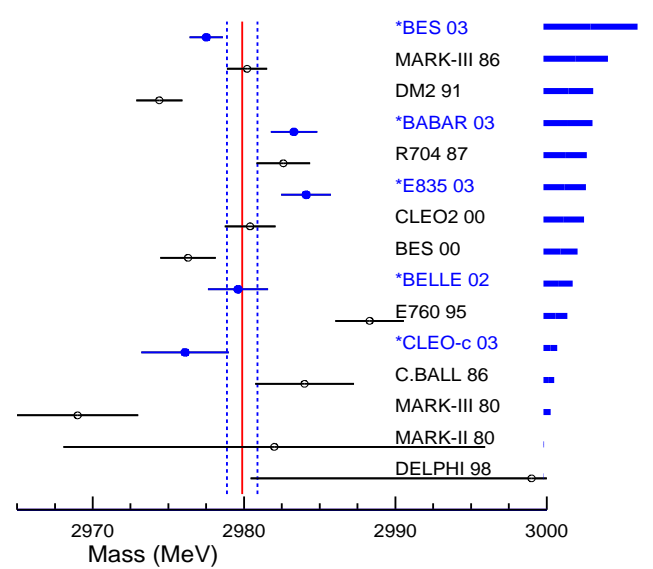

Figure 2. Measurements of the $\eta_{c}$ mass. The new measurements (BES 03, ${ }^{21}$ BABAR 03, ${ }^{22}$ E835 03, ${ }^{13}$ BELLE 02 ${ }^{23}$ and CLEO-c $03^{24}$ ) are indicated by a star. See PDG $2002^{20}$ for the references to the older measurements displayed here. The vertical bars indicate the average value (solid) and its error (dashed). The error includes the scale factor. The thick horizontal bars on the right give the relative weight of each experiment into the average value. 


\section{Charmonium Singlet States}

There are many new measurements related to the charmonium singlet states, in addition to the results from the $\bar{p} p$ experiments mentioned in the previous section. For example, there are 5 new measurements of the $\eta_{c}\left(1^{1} S_{0}\right)$ mass $^{13,21-24}$ (see Fig. 2). BES-II, ${ }^{21}$ which reconstructed the $\eta_{c}$ in 5 different decay modes in the $J / \psi$ data, achieved the smallest overall error. Consistency of the old and new measurements is questionable. A new preliminary measurement of the $\eta_{c}$ width by $\mathrm{BABAR}^{22}$ constitutes an even bigger experimental puzzle (see Figs. 3-4). The measurement error claimed by BABAR is much smaller than in any other measurement. At the same time the central value, $(33.3 \pm 2.5 \pm 0.5) \mathrm{MeV}$, is the highest ever measured; completely inconsistent with the previous world average value, ${ }^{20}\left(16.0_{-3.2}^{+3.6}\right) \mathrm{MeV}$.

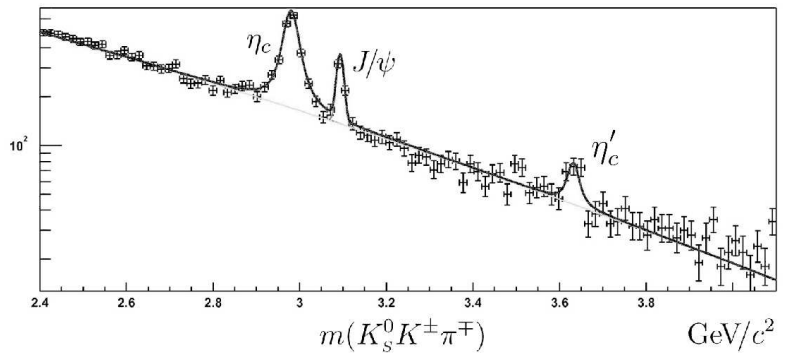

Figure 3. Observation of $\gamma \gamma \rightarrow \eta_{c}(1 S)$ and $\gamma \gamma \rightarrow \eta_{c}(2 S)$ by the BABAR experiment. ${ }^{22}$ A peak due to $e^{+} e^{-} \rightarrow \gamma J / \psi$ is also visible. These data serve as a precision determination of the $\eta_{c}(1 S)$ width and confirmation of the $\eta_{c}(2 S)$ state at its new mass value.

There have also been dramatic developments concerning the $\eta_{c}\left(2^{1} S_{0}\right)$ state. The Crystal Ball experiment at SPEAR claimed observation of this state over 20 years ago. ${ }^{15}$ They observed a peak in the inclusive photon energy spectrum from $\psi\left(2^{3} S_{1}\right)$ decays, which they interpreted as a magnetic dipole photon transition, $\psi(2 S) \rightarrow \gamma \eta_{c}(2 S) .{ }^{15}$ The Crystal Ball result corresponded to the hyperfine mass splitting in the radial excitation, which was only slightly smaller than in the ground state (92 MeV vs. 117 $\mathrm{MeV})$. Last year BELLE observed both $\eta_{c}$ states in $B \rightarrow K \eta_{c}(n S), \eta_{c}(n S) \rightarrow K_{s} K^{+} \pi^{-25}$ The $\eta_{c}(2 S)$ state appeared at much higher mass than in the Crystal Ball measurement, thus reducing the corresponding hyperfine splitting. BELLE also observed both $\eta_{c}$ states in the spectrum of the mass recoiling against $J / \psi$ in continuum $e^{+} e^{-}$annihilation to $J / \psi X{ }^{26}$ These results have been updated this year with larger statistics. ${ }^{27}$ The $\eta_{c}(2 S)$ mass obtained from these data differs by 1.9 standard deviations from the other BELLE result, but is still significantly higher than the Crystal Ball value.

$$
\begin{gathered}
\text { Average: }(25.0 \pm 3.3) \mathrm{MeV} \\
\mathrm{CL}=0.05 \% \text { Scale Factor }=1.8
\end{gathered}
$$

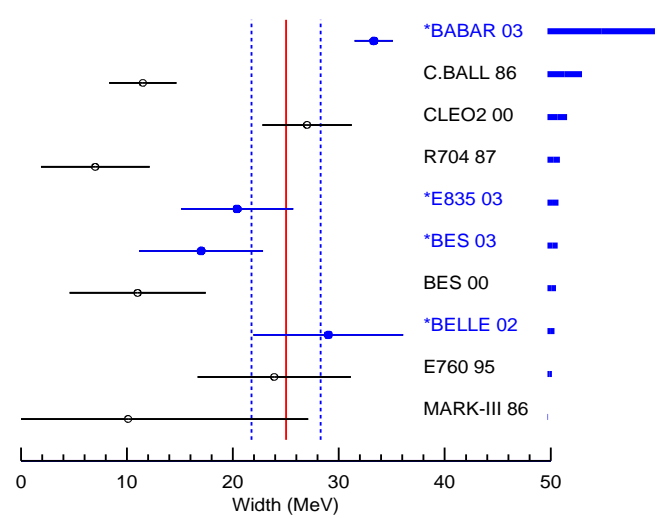

Figure 4. Measurements of the $\eta_{c}$ width. See Fig. 2 for references and explanation.

The production rate for charmonium pairs observed by BELLE in the latter analysis is surprisingly high. They also observe a large $e^{+} e^{-}$annihilation rate to $J / \psi$ plus open charm. They claim $(82 \pm 15 \pm 14) \%$ of prompt $J / \psi$ production $\left(P_{J / \psi}>2\right.$ $\mathrm{GeV})$ at $10.6 \mathrm{GeV}$ center-of-mass energy is associated with another $c \bar{c}$ pair in the event. ${ }^{27}$ This rate is an order of magnitude higher than that predicted theoretically, and constitutes the biggest puzzle in quarkonium production physics. ${ }^{28}$

This year the BABAR and CLEO experiments confirmed the $\eta_{c}(2 S)$ at its heavier mass by formation in the two-photon collision process ${ }^{22,29}$ (see Fig. 3). The discrepancy with the Crystal Ball mass measurement is resolved by the CLEO-c experiment, which has been able to remeasure the inclusive photon spectrum from the $\psi(2 S)$ for the first time since the Crystal Ball experiment (see the next section). While CLEO-c agrees well with the Crystal Ball on all other E1 and M1 transitions from the $\psi(2 S)$, the direct M1 transition to the $\eta_{c}(2 S)$ is not observed at the photon energy claimed by the Crystal Ball. The upper limit on the rate for this transition set by CLEO-c disagrees with the rate measured by the Crystal Ball. Therefore, the peak observed by Crystal Ball could not be due to $\eta_{c}(2 S)$ production.

Measurements of the $\eta_{c}(2 S)$ mass are summarized graphically in Fig. 5. All new mass measurements are consistent with each other. The to- 
tal width of this state, based on the BELLE ${ }^{25}$ and $\mathrm{BABAR}^{22}$ results, is $(19 \pm 10) \mathrm{MeV}$.

The new mass of the $\eta_{c}(2 S)$ state decreases the hyperfine mass splitting for the $2 S$-states by a factor of 2 compared to the old value. Since a wrong value prevailed for 20 years, it is interesting to check for an experimental bias on the phenomenological predictions for this splitting. A sample of potential model predictions for the ratio of the hyperfine mass splitting, $R_{H F} \equiv\left(M_{\psi(2 S)}-M_{\eta_{c}(2 S)}\right) /\left(M_{\psi(1 S)}-M_{\eta_{c}(1 S)}\right)$, is compared to the old $(R=0.79)$ and the new $\left(R_{H F}=0.412 \pm 0.028\right)$ experimental values in Fig. 6 . It appears that many old calculations were stretched to accommodate the old result, though there were a few that had the courage to contradict the data. The ratio of hyperfine mass splitting can be related to the ratio of the leptonic widths of the triplet states $\left(\Gamma_{e e}\right)$ using perturbative QCD. The new value agrees well with the prediction by Badalian and Bakker ${ }^{31}$ $R_{H F}=(0.48 \pm 0.07)$, based on this approach. Instead of using the measured values of $\Gamma_{e e}$, Recksiegel and Sumino ${ }^{32}$ extended the use of perturbative QCD to the extraction of the interquark potential at short distances relevant for the spin-spin forces. Their prediction for the hyperfine mass splitting ratio, $R_{H F}=0.42$, is in good agreement with the data, but the absolute values for the mass splittings are underestimated. Lattice QCD calculations ${ }^{33}$ predicted $R_{H F}=0.5$, not far from the measured value.

Average: (3637.7 \pm 4.4$) \mathrm{MeV}$ $\mathrm{CL}=14 \%$ Scale Factor $=1.3$

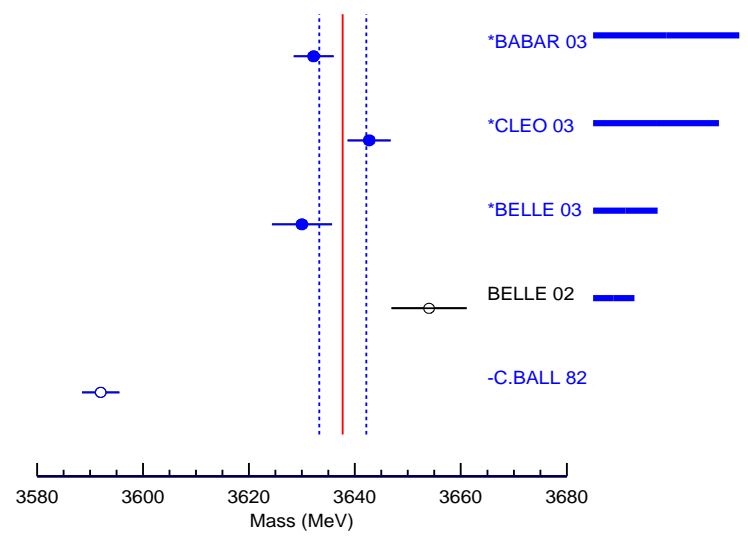

Figure 5. Measurements of the $\eta_{c}(2 S)$ mass (see the text for the references). The thick horizontal bars on the right give the relative weight of each experiment into the average value. The Crystal Ball measurement was excluded from the average.

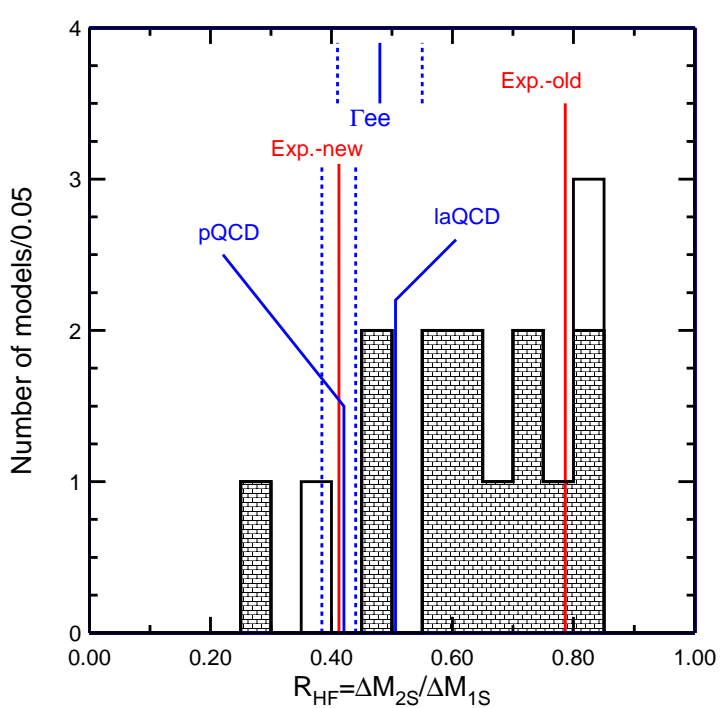

Figure 6. Measurements and predictions for the ratio of the hyperfine mass splittings in the $2 S$ and $1 S$ states in charmonium. The old and new experimental values are indicated by vertical bars. The dashed lines around the new value show the experimental error. The histogram shows predictions of a sample of potential models. ${ }^{30}$ Only models which gave a good fit to the masses of all known charmonium states were included. The shaded part of the histogram represents the models published before the new measurements of the $\eta_{c}(2 S)$ mass. Values predicted by scaling from the measured leptonic widths ratio ${ }^{31}\left(\Gamma_{e e}\right)$, by perturbative QCD alone ${ }^{32}$ (pQCD) and by lattice $\mathrm{QCD}^{33}$ (laQCD) are also indicated.

\section{Radiative Transitions}

Since sizes of heavy quarkonia are small or comparable to the wavelengths of transition photons, selection rules are given by multipole expansion. As the system is non-relativistic, electric dipole transitions $(\Delta L=1, \Delta S=0)$ are much stronger than magnetic dipole transitions $(\Delta L=0, \Delta S=1)$. The latest improvements in measurements of these transitions come mostly from the CLEO experiment, which is equipped with an excellent $\mathrm{CsI}(\mathrm{Tl})$ calorimeter. Recently, after increasing statistics of the $\Upsilon(1,2,3 S)$ data samples by an order of magnitude, CLEO turned the beam energy down and collected also $\psi(2 S)$ data. This was the beginning of the CLEO-c phase. Inclusive photon spectra collected at $\psi(2 S)$ and $\Upsilon(2 S)$ by CLEO are compared in Fig. 7. The dominant peaks are due to E1 transitions, $2^{3} S_{1} \rightarrow \gamma 1^{3} P_{J}$. The peaks in bottomonium system appear smaller because of the increased $\pi^{0} \rightarrow \gamma \gamma$ background induced by higher particle multiplicities. The peaks are more crowded together reflecting the smaller fine structure of the $1^{3} P_{J}$ states 
in the more non-relativistic $\Upsilon$ system. The E1 cascade lines, $1^{3} P_{J} \rightarrow \gamma 1^{3} S_{1}$ are also visible. The charmonium data also reveal a small peak due to the hindered M1 transition, $2^{3} S_{1} \rightarrow \gamma 1^{1} S_{0}$. This is the first confirmation of this transition since the original observation by the Crystal Ball. As discussed in the previous section, the direct M1 transition, $2^{3} S_{1} \rightarrow \gamma 2{ }^{1} S_{0}$, is ruled out for the photon energy, $\eta_{c}(2 S)$ width and rate claimed by the Crystal Ball. The preliminary CLEO-c results ${ }^{45}$ for the $\psi(2 S)$ photon lines are: $\mathcal{B}\left(\psi\left(2^{3} S_{1}\right) \rightarrow \gamma \chi_{c}\left(1^{3} P_{J}\right)\right)=\{9.75 \pm$ $0.14 \pm 1.17,9.64 \pm 0.11 \pm 0.69,9.83 \pm 0.13 \pm 0.87\} \%$ for $J=\{2,1,0\}, \mathcal{B}\left(\psi\left(2^{3} S_{1}\right) \rightarrow \gamma \eta_{c}\left(1^{1} S_{0}\right)\right)=(0.278 \pm$ $0.033 \pm 0.049) \%$ and $\mathcal{B}\left(\psi\left(2^{3} S_{1}\right) \rightarrow \gamma \eta_{c}\left(2^{1} S_{0}\right)\right)<$ $0.2 \%$ for $E_{\gamma}=(91 \pm 5) \mathrm{MeV}$ and $\Gamma\left(\eta_{c}(2 S)\right)=8$ $\mathrm{MeV}(90 \%$ CL).
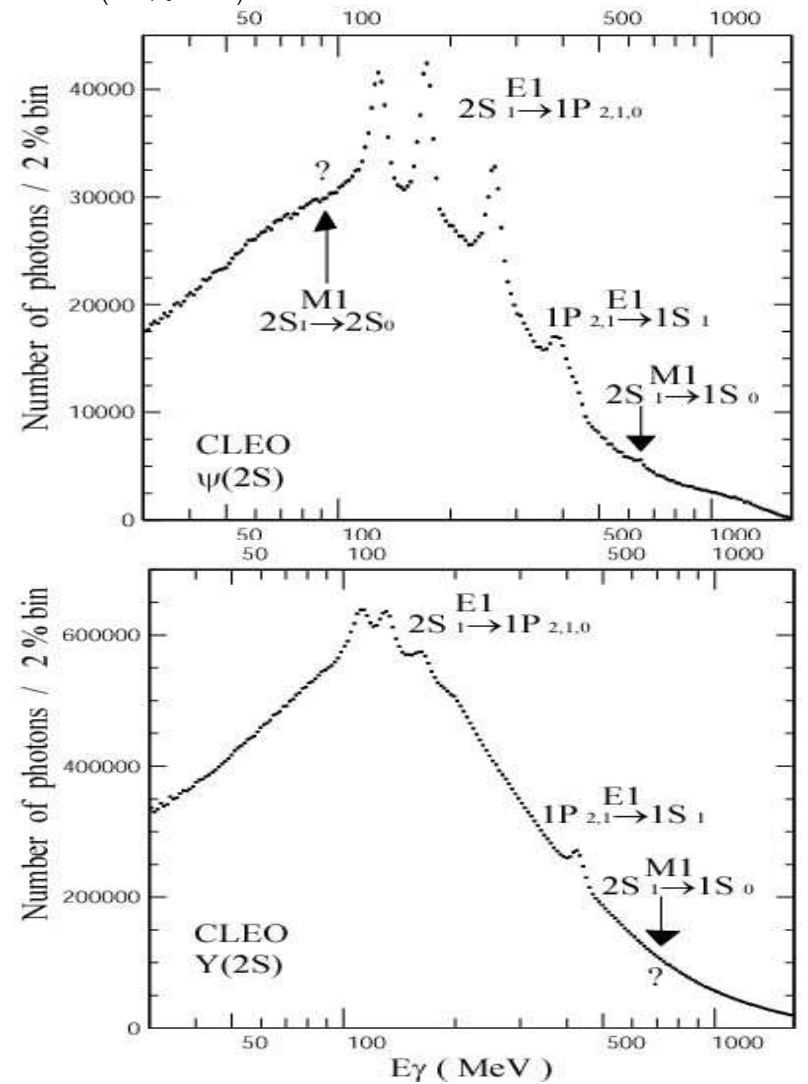

Figure 7. Inclusive photon spectrum from $2^{3} S_{1}$ decays in the $c \bar{c}$ (top) and $b \bar{b}$ (bottom) systems measured with the CLEO detector. The data correspond to about $1.5 \mathrm{M} \psi(2 S)$ and $9 \mathrm{M}$ $\Upsilon(2 S)$ decays.

None of M1 transitions, hindered or direct, are observed for the bottomonium. This is not a big surprise, since the backgrounds are much higher and the expected branching ratios are smaller in the more non-relativistic $b \bar{b}$ system. Searches for the singlet $\eta_{b}$ state in two-photon collisions at LEP has also yielded upper limits only, ${ }^{34}$ thus no singlet states have been observed in bottomonium so far. The M1 rate measurements can be translated into values of the corresponding matrix elements, which are compared to theoretical predictions in Fig. 8. The matrix elements for the hindered M1 transitions are expected to be very small, since they are generated by relativistic and finite size corrections. Therefore, they are difficult to predict. Only very recent calculations are consistent with all charmonium and bottomonium data. Similar comparisons for the E1 matrix elements is shown in Fig. 9. Non-relativistic calculations (hollow circles) overestimate the E1 rates in charmonium. The predictions with relativistic corrections (filled triangles) can reproduce the data. Relativistic effects in the dominant E1 transitions in bottomonium are much smaller. However, relativistic calculations are needed to reproduce the rare $3^{3} S_{1} \rightarrow \gamma 1^{3} P_{J}$ transition rate. This matrix element is small because of the cancellations in the integral for the E1 operator between the initial and the final state wave functions ${ }^{39}$ for which the number of nodes differ by two.

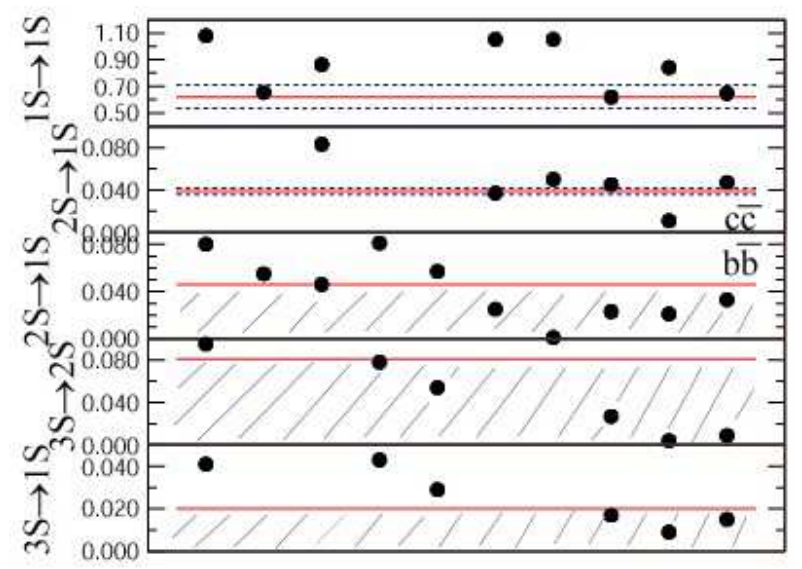

Figure 8. Measured and predicted values of matrix elements for M1 transitions in heavy quarkonia. The measured values are calculated from the world average branching ratios and total widths of the triplet $S$ states. The central value and error bars for the $c \bar{c}$ data are indicated with solid and dashed lines respectively. For the $b \bar{b}$ data, allowed ranges from the preliminary CLEO analysis ${ }^{45}$ are shaded (90\% CL). The theoretical predictions $^{35}$ (points) are ordered according to the publication date. 


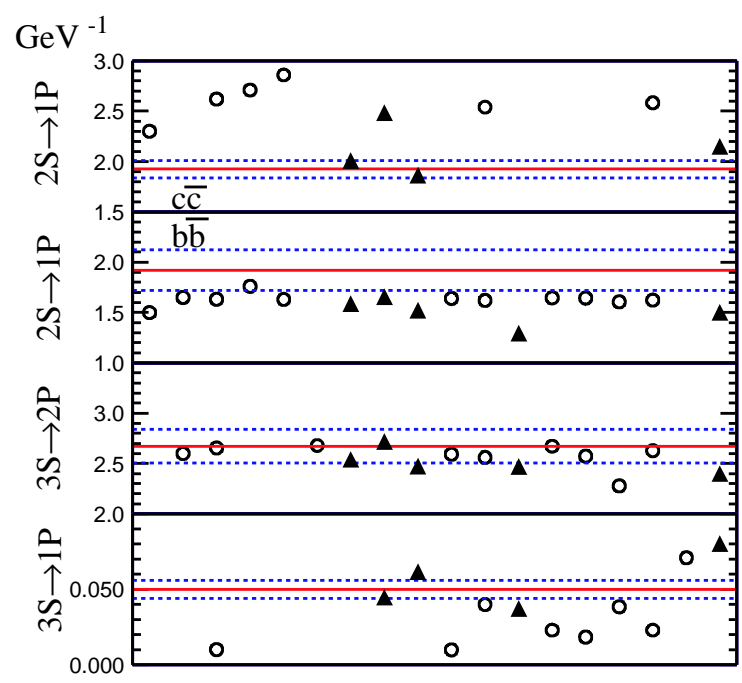

Figure 9. Measured and predicted values of matrix elements for E1 transitions in heavy quarkonia averaged over different spins of the triplet $P$ states. The measured values are calculated from the world average radiative branching ratios and total widths of the triplet $S$ states. The central values and error bars for the measured values are indicated with solid and dashed lines respectively. Circles (triangles) show nonrelativistic (relativistic) calculations. The relativistic calculations are averaged over spins with the same weights as the data. The predictions ${ }^{36}$ are ordered according to the publication date.

\section{Hadronic Transitions}

Heavy quarkonia can also change excitation levels by emission of soft gluons, that turn into light hadrons. The multipole expansion approach has proven to be useful also for hadronic transitions ${ }^{37,38}$ explaining their gross characteristics. The most prominent are $\pi \pi$ transitions among $n^{3} S_{1}$ states, which can be mediated by two-gluon emission of the E1-E1 type. In fact, these are dominant decays for both $\psi(2 S)$ and $\Upsilon(2 S)$. The ratio of the measured widths for the these transitions agrees with a suppression by about a factor of 10 predicted by the multipole expansion mode $^{38}$ due to the smaller size of the $b \bar{b}$ system. The multipole expansion model is also able to explain the $M(\pi \pi)$ distributions for these transitions, which follow the same pattern and peak at high values. Dipion transitions from $\Upsilon(3 S)$ to $\Upsilon(2 S)$ and $\Upsilon(1 S)$ are observed too. They have smaller rates either due to the phase space suppressions or cancellations in the dipole matrix element integral for the $3 S \rightarrow \pi \pi 1 S$ transition. The $M(\pi \pi)$ distribution peaks at low and high mass values for the latter transition, which reveals some dynamics beyond the multipole expansion approach. In charmonium, an $\eta$ transition has been observed between the triplet $2 S$ and $1 S$ states. ${ }^{20}$ This transition is of a magnetic type (E1·M2), thus it has a smaller rate than the $\pi \pi$ transition. A $\pi^{0}$ transition is also observed, but with a tiny rate due to isospin violation. None of these transitions are observed between the $\Upsilon$ states ${ }^{45}$ which is not surprising since there is an additional suppression by at least a factor $1 / m_{Q}^{2}$ for this type of transition.

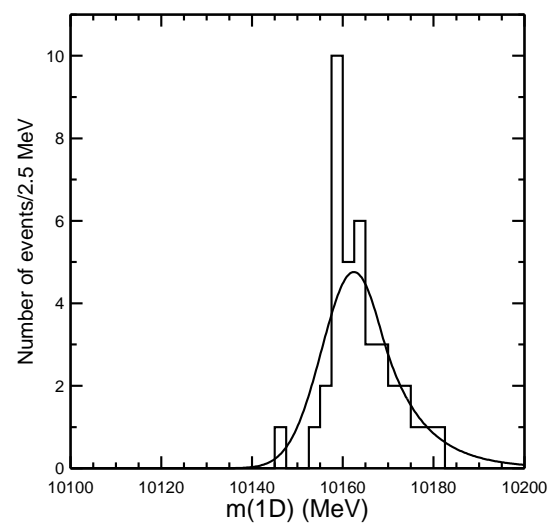

Figure 10. Recoil mass against the lowest energy photons in four-photon cascade between the $\Upsilon(3 S)$ and $\Upsilon(1 S)$, consistent with $\chi_{b}\left(2 P_{2,1}\right)$ and $\chi_{b}\left(1 P_{2,1}\right)$ as intermediate states, as observed by CLEO. The cascades via the $\Upsilon(2 S)$ state are suppressed, thus the remaining events are $\Upsilon(1 D)$ candidates. The recoil mass is a measure of the $\Upsilon(1 D)$ mass. The fit to the data (solid line) assumes production of just one $\Upsilon(1 D)$ state, with a natural width much smaller than the experimental resolution. The observed events are most likely dominated by the production of the $\Upsilon\left(1 D_{2}\right)$ state. The preliminary CLEO results give $(10161.1 \pm 0.6 \pm 1.6) \mathrm{MeV}$ for the mass of this state.

The transitions among triplet $n S$ states discussed above were first observed over 20 years ago. A large number of other hadronic transitions are possible, ${ }^{39}$ especially for the $b \bar{b}$ system which has a large number of long-lived states. Such transitions had not been observed until recently, when CLEO reported the first observation of $\chi_{b}\left(2^{3} P_{2,1}\right) \rightarrow \omega \Upsilon(1 S)$ transitions. ${ }^{40}$ The phase-space for these transitions is so small, that this decay is forbidden for the $\chi_{b}\left(2^{3} P_{0}\right)$. The measured branching ratios for the $\chi_{b}\left(2^{3} P_{1}\right)$ and $\chi_{b}\left(2^{3} P_{2}\right)$ states are of the order of a couple of percent $((1.6 \pm 0.3 \pm 0.2) \%$ and $(1.1 \pm 0.3 \pm$ $0.1) \%$ respectively), in spite of the phase space suppression, which reveals that the underlying transition is quite strong. In fact, this transition is of chromoelectric type, E1·E1·E1 (three gluons are needed to generate it). Voloshin ${ }^{41}$ pointed out that since the matrix element does not depend on the spin of the $2 P_{J}$ state, transition branching ratios for $J=1$ and $J=2$ should be comparable (as the phase space 
factors approximately cancel the effect due to the smaller total width of the $J=1$ state). The data are consistent with this prediction.

As hadronization probabilities are difficult to estimate, uncertainties in absolute rate predictions for hadronic transitions are usually very large. When resonances dominate the transition, there are often no theoretical predictions for the rate. For example, there are no rate estimates for the $\omega$ transition discussed above. Predictions for other yet unobserved types of transitions vary by orders of magnitude. The predictions based on a model developed by $\mathrm{Yan}^{38}$ and Kuang ${ }^{42}$ tend to be much larger than the predictions based on a different approach introduced by Voloshin, Zakharov, Novikov and Shifman. ${ }^{43}$

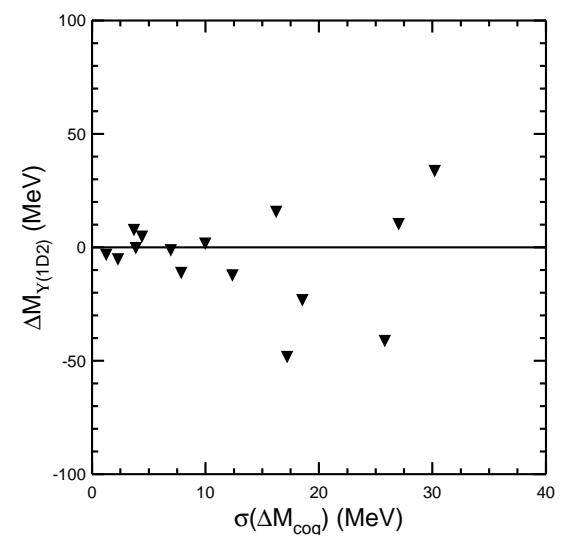

Figure 11. The difference between the predicted and the measured $\Upsilon\left(1^{3} D_{2}\right)$ mass (vertical axis) versus overall quality of the potential model expressed as the r.m.s. of the differences for the center-of-gravity masses of long-lived $b \bar{b}$ states (horizontal axis). Predictions for the $1 \mathrm{~S}, 2 \mathrm{~S}, 3 \mathrm{~S}, 1 \mathrm{P}, 2 \mathrm{P}$ and $1 \mathrm{D}$ masses are included in the r.m.s. calculation for all models displayed here. ${ }^{46}$

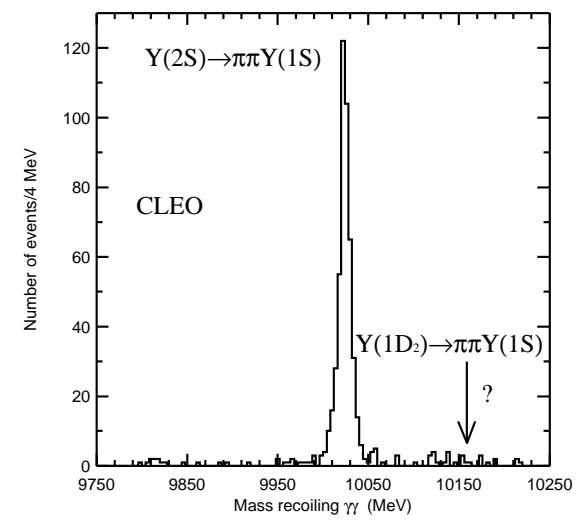

Figure 12. Search for $\Upsilon\left(1^{3} D_{2}\right) \rightarrow \pi^{+} \pi^{-} \Upsilon(1 S)$ transitions. Recoil mass against two photons in $\gamma \gamma \pi^{+} \pi^{-} l^{+} l^{-}$events is plotted. The expected signal position is indicated by the arrow.

One suitable place to test various predictions are dipion transitions from $1^{3} D_{J}$ to $1^{3} S_{1}$. Last year the $\Upsilon\left(1^{3} D_{J}\right)$ states were discovered by CLEO in the four-photon cascade: ${ }^{44} \Upsilon\left(3^{3} S_{1}\right) \rightarrow \gamma \chi_{b}\left(2^{3} P_{J_{2}}\right)$, $\chi_{b}\left(2^{3} P_{J_{2}}\right) \rightarrow \gamma \Upsilon\left(1^{3} D_{J_{D}}\right), \Upsilon\left(1^{3} D_{J_{D}}\right) \rightarrow \gamma \chi_{b}\left(1^{3} P_{J_{1}}\right)$, $\chi_{b}\left(1^{3} P_{J_{1}}\right) \rightarrow \gamma \Upsilon\left(1^{3} S_{1}\right)$ followed by $\Upsilon\left(1^{3} S_{1}\right) \rightarrow l^{+} l^{-}$. CLEO has analyzed more data since then. The recoil mass distribution against the first two photons in the cascade is consistent with the detector resolution (Fig. 10). Thus the data are dominated by production of just one $\Upsilon\left(1^{3} D_{J_{D}}\right)$ state. Theoretically, production of the $J_{D}=2$ state is expected to dominate. This spin assignment is also favored by the experimental data. ${ }^{44}$ The measured mass fits the predictions of the potential models well, especially those which provide a good fit to the other narrow $b \bar{b}$ states. This is illustrated in Fig. 11. The lattice QCD calculations also reproduce the observed mass very well. ${ }^{2}$ CLEO determines ${ }^{45}$ the four-photon cascade branching ratio to be $(2.6 \pm 0.5 \pm 0.5) \times 10^{-5}$. Within the errors this rate is consistent with the predictions by Godfrey and Rosner, ${ }^{47} 3.8 \times 10^{-5}$ $\left(2.6 \times 10^{-5}\right.$ for $J_{D}=2$ alone $)$, based on the E1 matrix elements and estimates of hadronic widths of the $\Upsilon\left(1^{3} D_{J_{D}}\right)$ and $\chi_{b}\left(2^{3} P_{J_{2}}\right)$ states. CLEO also looked for $\Upsilon\left(1^{3} D_{J_{D}}\right) \rightarrow \pi^{+} \pi^{-} \Upsilon\left(1^{3} S_{1}\right)$ replacing the third and fourth photons in the cascade with a $\pi^{+} \pi^{-}$pair. The recoil mass against the remaining two photons in the cascade is plotted in Fig. 12. Since no signal is found, upper limits on the product of branching ratios are set. They are compared in Table 2 to the theoretical predictions calculated by Rosner ${ }^{48}$ using predictions for $\Upsilon\left(1^{3} D_{J_{D}}\right) \rightarrow \pi^{+} \pi^{-} \Upsilon\left(1^{3} S_{1}\right)$ width by various authors ${ }^{42,49,50}$ together with the E1photon matrix elements and estimates of hadronic widths of the $\Upsilon\left(1^{3} D_{J_{D}}\right)$ and $\chi_{b}\left(2^{3} P_{J_{2}}\right)$ states. Large $\Upsilon\left(1^{3} D_{J_{D}}\right) \rightarrow \pi^{+} \pi^{-} \Upsilon\left(1^{3} S_{1}\right)$ widths, as predicted by Kuang-Yan, ${ }^{42}$ are ruled out. Better experimental sensitivity is needed to test the other models. Voloshin pointed out ${ }^{51}$ that $\Upsilon\left(1^{3} D_{J_{D}}\right) \rightarrow \eta \Upsilon\left(1^{3} S_{1}\right)$ transition may be of comparable strength to the $\pi \pi$ transition between these states. CLEO doesn't find this transition either and sets a preliminary upper limit of $\mathcal{B}\left(\Upsilon\left(3^{3} S_{1}\right) \rightarrow \gamma \chi_{b}\left(2^{3} P_{J_{2}}\right)\right) \mathcal{B}\left(\chi_{b}\left(2^{3} P_{J_{2}}\right) \rightarrow\right.$ $\left.\gamma \Upsilon\left(1^{3} D_{J_{D}}\right)\right) \mathcal{B}\left(\Upsilon\left(1^{3} D_{J_{D}}\right) \rightarrow \eta \Upsilon\left(1^{3} S_{1}\right)\right)<2.3 \times 10^{-4}$ at $90 \% \mathrm{CL}$.

There are also new results on $1^{3} D_{1} \rightarrow \pi^{+} \pi^{-} 1^{3} S_{1}$ transitions in the charmonium system. Here, theoretical situation is complicated by mixing of the $\psi\left(1^{3} D_{1}\right)$ state with the $\psi\left(2^{3} S_{1}\right)$. The observed state, $\psi(3770)$, is also above the open flavor threshold, 
Table 2. Theoretical predictions and preliminary CLEO upper limits (at $90 \% \mathrm{CL})$ on $\mathcal{B}\left(\Upsilon\left(3^{3} S_{1}\right) \rightarrow \gamma \chi_{b}\left(2^{3} P_{J_{2}}\right)\right) \mathcal{B}\left(\chi_{b}\right.$ $\left.\left(2^{3} P_{J_{2}}\right) \rightarrow \gamma \Upsilon\left(1^{3} D_{J_{D}}\right)\right) \mathcal{B}\left(\Upsilon\left(1^{3} D_{J_{D}}\right) \rightarrow \pi^{+} \pi^{-} \Upsilon\left(1^{3} S_{1}\right)\right)$ in units of $10^{-4}$. The theoretical predictions come from the paper by Rosner.48 The first row corresponds to the cascade via the $J_{D}=2$ state observed in the four-photon cascade by CLEO. The second row corresponds to any $\Upsilon\left(1^{3} D_{J_{D}}\right)$ state with mass in the $10140-10180 \mathrm{MeV}$ interval.

\begin{tabular}{|l|r|r|r|r|}
\hline & CLEO & \multicolumn{3}{|c|}{$\Gamma_{\pi \pi}$ model } \\
\cline { 3 - 5 } & & Kuang-Yan & Moxhay & Ko $^{40}$ \\
\hline$\Upsilon\left(1^{3} D_{2}\right)$ & $<1.1$ & 9.2 & 0.049 & 0.39 \\
$\Upsilon\left(1^{3} D_{J_{D}}\right)$ & $<2.7$ & 17.7 & 0.094 & 0.75 \\
\hline
\end{tabular}

therefore it has a large width for decay to $D \bar{D}$ which suppresses branching ratios for any transitions to the other charmonium states. BES claims observation of such a transition in the data consisting of $5.7 \times 10^{4} \psi(3770)$ decays. ${ }^{52}$ Their reconstruction efficiency is $17 \%$. They observe 9 signal candidates with an estimated background of $2.2 \pm 0.4$ events (see Fig. 13a). The corresponding branching ratio is $(0.59 \pm 0.26 \pm 0.16) \%$. Such a large branching ratio would favor the Kuang-Yan model ${ }^{53}$ contrary to the $b \bar{b}$ results discussed above. CLEO-c has also analyzed their first $\psi(3770)$ sample. ${ }^{45}$ They have a smaller sample of $\psi(3770)$ decays $\left(4.5 \times 10^{4}\right)$ but larger reconstruction efficiency $(37 \%)$. They have only 2 events in the signal region, consistent with the estimated background level (see Fig. 13b). They set a preliminary upper limit $\mathcal{B}\left(\psi(3770) \rightarrow \pi^{+} \pi^{-} J / \psi\right)<0.26 \%$ (90\% CL). This result is not inconsistent with the BES value because of the large experimental errors in both measurements. However, the CLEO result indicates that it is premature to favor the KuangYan model on the basis of the $\psi(3770)$ data. BES is already analyzing a larger data sample and CLEO is expected to increase their statistics by an order of magnitude this fall, thus more accurate results are expected next year.

\section{New Particle Discovered by BELLE}

Decays to $\pi^{+} \pi^{-} J / \psi$ are also a way to search for other charmonium states, for example $h_{c}\left(1^{1} P_{1}\right), \psi\left(1^{3} D_{2}\right)$, $\psi\left(1^{1} D_{2}\right)$ etc.. Many of these states cannot be directly formed in $e^{+} e^{-}$annihilation, however they can be produced in $\bar{p} p$ annihilation, $B$ decays or fragmentation processes. BELLE inspected resonance structures in the $\pi^{+} \pi^{-} J / \psi$ system produced in the decay $B^{ \pm} \rightarrow K^{ \pm}\left(\pi^{+} \pi^{-} J / \psi\right), J / \psi \rightarrow l^{+} l^{-}$. The distribution of $\Delta M=M\left(\pi^{+} \pi^{-} J / \psi\right)-M(J / \psi)$ observed

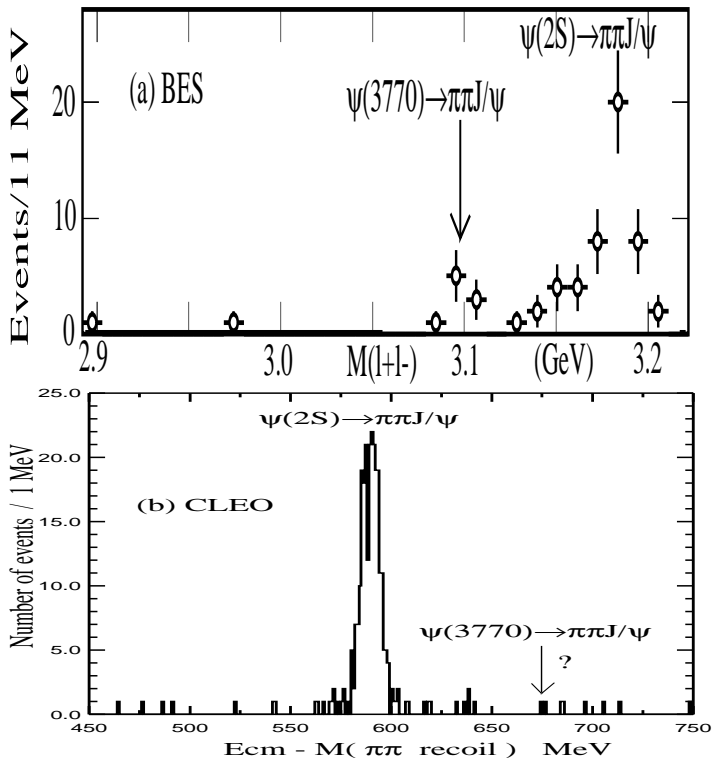

Figure 13. Searches for $\psi(3770) \rightarrow \pi^{+} \pi^{-} J / \psi$ transitions. The expected signal positions are indicated with arrows. The dominant peak in each distribution is due to $e^{+} e^{-} \rightarrow \gamma \psi(2 S)$, $\psi(2 S) \rightarrow \pi^{+} \pi^{-} J / \psi$. (a) BES results. $M\left(l^{+} l^{-}\right)$mass for kinematically constrained $\pi^{+} \pi^{-} l^{+} l^{-}$events is plotted. The signal is expected at the $J / \psi$ mass. (b) CLEO results. The difference between the center-of-mass energy and the recoil mass against the $\pi^{+} \pi^{-}$pair is plotted with no kinematic constraints, but after the cut on $M\left(l^{+} l^{-}\right)$around the $J / \psi$ mass. The signal is expected at the mass difference between the $\psi(3770)$ and the $J / \psi$.

by BELLE $^{54}$ in a sample of $3 \times 10^{8} B$ mesons is shown in Fig. 14. The prominent peak is due to the $\psi\left(2^{3} S_{1}\right)$. There is also a smaller but very significant peak observed at a higher mass. By performing an unbinned maximum likelihood fit to $\Delta M$, the beamconstraint $B$ meson mass and energy difference between the $B$ candidate and the beam energy BELLE finds $35.7 \pm 6.8$ events in the second peak with a statistical significance of 10.3 standard deviations. The mass determined from the $\Delta M$ peak position is $(3872.0 \pm 0.6 \pm 0.5) \mathrm{MeV}$. The observed width of the peak is consistent with the detector resolution, therefore the new state is long-lived $\left(\Gamma_{t o t}<2.3 \mathrm{MeV}\right.$ at $90 \% \mathrm{CL})$.

The invariant mass of the $\pi^{+} \pi^{-}$system for the signal events is strongly peaked at high mass values. The peaking is stronger than predicted by $\mathrm{Yan}^{38}$ with the multipole expansion approach for $S \rightarrow S$ transitions, and much stronger than predicted for $D \rightarrow S$ transitions in the quarkonium system, as illustrated in Fig. 15. The observed dipion mass distribution is suggestive of the isospin violating $X(3872) \rightarrow$ $\rho^{0} J / \psi$ process instead. Isospin violation can be experimentally verified by measuring $\Gamma(X(3872) \rightarrow$ 
$\left.\pi^{0} \pi^{0} J / \psi\right) / \Gamma\left(X(3872) \rightarrow \pi^{+} \pi^{-} J / \psi\right){ }^{55,56}$ For isospin conserving $\pi \pi$ transitions, this ratio should be approximately $1 / 2$, whereas $\rho^{0}$ does not decay to $\pi^{0} \pi^{0}$.

Soon after BELLE's announcement at the Lepton-Photon Symposium, the $X(3872)$ particle was confirmed by CDF in $\pi^{+} \pi^{-} J / \psi, J / \psi \rightarrow \mu^{+} \mu^{-57}$ In $220 \mathrm{pb}^{-1}$ of Run-II data they observe $704 \pm 67$ signal events. The preliminary mass measurement, $3871.4 \pm 0.7 \pm 0.4 \mathrm{MeV}$, is consistent with BELLE's result. Their data also show peaking at high dipion mass.

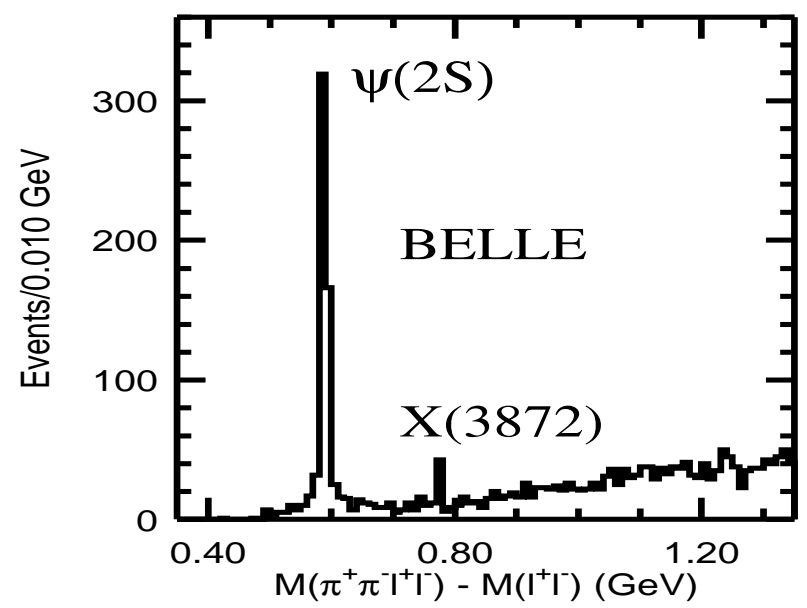

Figure 14. Distribution of $M\left(\pi^{+} \pi^{-} l^{+} l^{-}\right)-M\left(l^{+} l^{-}\right)$as observed by BELLE for $B$ decay candidates. ${ }^{54}$ The first peak corresponds to the $\psi(2 S)$ resonances. The second peak represents the $X(3872)$ particle.

Perhaps the most puzzling property of the newly discovered particle is its mass. Within the experimental errors it coincides with the sum of $D^{0}$ and $D^{* 0}$ masses: $m_{X}-m_{D^{0}}-m_{D^{* 0}}=0.2 \pm 0.9 \mathrm{MeV}$ (using the average of the BELLE and CDF results). One exciting interpretation, which actually predicts this to be the case, is a $D \bar{D}^{*}\left(+\bar{D} D^{*}\right)$ molecule. Constituent mesons would likely be in a relative Swave creating a $J^{P}=1^{+}$state. "Molecular charmonium" was first discussed in the literature in the mid-seventies ${ }^{58}$ and was initially introduced to explain the complicated coupling of the $e^{+} e^{-}$resonances above the open flavor threshold to various decay modes involving $D$ and $D^{*}$ meson pairs. A satisfactory description of the $e^{+} e^{-}$data was later achieved within a simple $c \bar{c}$ bound state model. ${ }^{59,60}$ However, since meson molecules were also proposed in the context of light hadron spectroscopy ${ }^{61}$ the concept of molecular charmonium did not go away. Interactions in the $D \bar{D}^{*}$ system (also $B \bar{B}^{*}$ ) were found to be attractive when described by the pion- exchange force. ${ }^{62,63}$ No such force would exist in the $D \bar{D}$ (or $B \bar{B}$ ) system. Quantitative estimates showed that the $D \bar{D}^{*}$ system could be only loosely bound if at all, with better prospects for the $B \bar{B}^{*}$ molecule. Tornqvist showed that in the limit of isospin symmetry only an isoscalar molecule would be bound. ${ }^{62}$ Isospin is expected to be broken since the binding energy is comparable to the isospin mass splittings (the $D^{+} D^{-*}$ threshold is $8 \mathrm{MeV}$ above the $D^{0} \bar{D}^{0 *}$ threshold). Close and Page ${ }^{64}$ argued that the isospin breaking does not change the conclusion that there is only one molecular system expected near the $D \bar{D}^{*}$ threshold. The loose binding makes it difficult for the molecule to rearrange the quark content in the meson subcomponents, thus strong decays to a charmonium state plus light hadrons are expected to have small widths. Decays to $\pi^{+} \pi^{-} J / \psi$ could proceed via the isospin violating $\rho^{0} J / \psi$ channel consistent with the BELLE's dipion mass distribution. The molecular interpretation predicts that decays to $D^{0} \bar{D}^{0} \pi^{0}$ and $D^{0} \bar{D}^{0} \gamma$ should occur, since the $D^{0 *}$ is almost on shell. The ratio of the widths for these decays should be approximately $3: 2$ and their sum $60-100$ $\mathrm{keV}^{62,64,65,56}$ Therefore, the molecular model is consistent with the narrow width of the observed state.

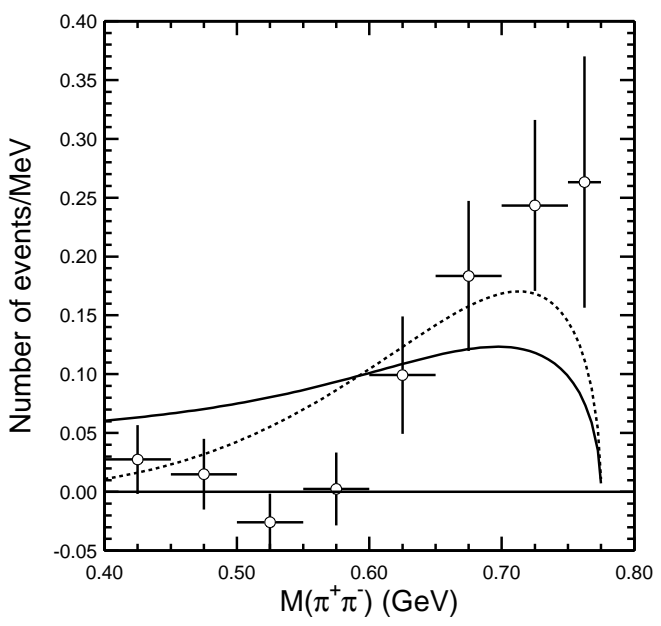

Figure 15. Dipion mass distribution in BELLE's data ${ }^{66}$ for $X(3872) \rightarrow \pi^{+} \pi^{-} J / \psi$. The data are compared to the theoretical shapes predicted by $\operatorname{Yan}^{38}$ for $D \rightarrow S$ (solid line) and $S \rightarrow S$ (dashed line) transitions in the quarkonium system. The distributions were normalized to the same number of events. The efficiency dependence on $M\left(\pi^{+} \pi^{-}\right)$has been neglected in the comparison.

Traditional charmonium states can also be narrow at $3872 \mathrm{MeV}$ if they cannot decay to $D \bar{D}$. Possible candidates, ordered according to increasing mass predictions are $\psi\left(1^{3} D_{2^{--}}\right), \eta_{c 2}\left(1^{1} D_{2^{-+}}\right)$, 
$\chi_{c}\left(2^{3} P_{1^{++}}\right), h_{c}\left(2^{1} P_{1^{+-}}\right)$and $\eta_{c}\left(3^{1} S_{0^{-+}}\right)$.

The lower excitations of the $\eta_{c}$ are already broader than the upper limit on the natural width of the new state, thus we can discard the $\eta_{c}\left(3{ }^{1} S_{0^{-+}}\right)$ possibility. The other listed states can satisfy this upper limit. For the remaining options, radiative E1 transitions to the other charmonium states should dominate over $\pi \pi J / \psi$. This is especially true for the singlet states, for which the dipion transition would involve chromomagnetic interactions (spinflip). Since the $h_{c}\left(1^{1} P_{1^{+-}}\right)$has not been seen in $B$ decays, the $h_{c}\left(2^{1} P_{1^{+-}}\right)$hypothesis is unlikely.

BELLE has also just observed $B^{ \pm} \rightarrow$ $K^{ \pm} \psi(3770)(\psi(3770) \rightarrow D \bar{D})$ for the first time. ${ }^{67}$ The measured branching ratio, $(4.8 \pm 1.1 \pm 1.2) \times 10^{-4}$, is comparable to ${ }^{20} \mathcal{B}\left(B^{ \pm} \rightarrow K^{ \pm} \psi(2 S)\right)=(6.8 \pm$ $0.4) \times 10^{-4}$. Theoretically, $\mathcal{B}\left(B^{ \pm} \rightarrow K^{ \pm} \psi\left(1^{3} D_{2}\right)\right)$ is expected to be 1.6 times larger than the production of $\psi(3770)$ (assumed to be $\left.\psi\left(1^{3} D_{1}\right)\right) .{ }^{68}$ Together, with BELLE's result, ${ }^{54} \mathcal{B}\left(B^{ \pm} \rightarrow K^{ \pm} X\right) \mathcal{B}(X \rightarrow$ $\left.\pi^{+} \pi^{-} J / \psi\right)=(0.063 \pm 0.012 \pm 0.007) \times \mathcal{B}\left(B^{ \pm} \rightarrow\right.$ $\left.K^{ \pm} \psi(2 S)\right) \mathcal{B}\left(\psi(2 S) \rightarrow \pi^{+} \pi^{-} J / \psi\right)$, the $\psi\left(1^{3} D_{2}\right)$ interpretation requires $\mathcal{B}\left(\psi\left(1^{3} D_{2}\right) \rightarrow \pi^{+} \pi^{-} J / \psi\right)$ to be about 1 to $3 \%$, much smaller than the naïve expectations ${ }^{69,56}$ for the dominant radiative decay of this state, $\mathcal{B}\left(\psi\left(1^{3} D_{2}\right) \rightarrow \gamma \chi_{c}\left(1^{3} P_{1}\right)\right)>50 \%$. Also, as discussed in the previous section the $\Upsilon\left(1^{3} D_{2}\right)$ state was observed via its radiative decay, whereas only an upper limit on the decay to $\pi^{+} \pi^{-} \Upsilon$ exists. However, BELLE observes no evidence for the photon transitions to $\chi_{c}\left(1^{3} P_{1}\right)$ and sets the following 90\% CL limit: $\mathcal{B}\left(X(3872) \rightarrow \gamma \chi_{c}\left(1^{3} P_{1}\right)\right) /$ $\mathcal{B}\left(X(3872) \rightarrow \pi^{+} \pi^{-} J / \psi\left(1^{3} S_{1}\right)\right)<0.89$. Coupled channel effects can be big (proximity of the $D \bar{D}^{*}$ threshold!) and significantly alter the naïve predictions for the radiative widths. ${ }^{70}$ Quantitative estimates are needed.

It will also be useful to resolve the experimental controversy about $\mathcal{B}\left(\psi(3770) \rightarrow \pi^{+} \pi^{-} J / \psi\right)$, since the width for $\psi\left(1^{3} D_{2}\right) \rightarrow \pi^{+} \pi^{-} J / \psi$ is related to the width for $\psi\left(1^{3} D_{1}\right) \rightarrow \pi^{+} \pi^{-} J / \psi$. The CLEO-c upper limit on the $\psi(3770) \rightarrow \pi^{+} \pi^{-} J / \psi$ branching ratio (see the previous section) suggests that the latter is not necessarily much larger than the value induced by the $2^{3} S_{1}-1^{3} D_{1}$ mixing, while the BES measurement indicates a rather large rate for direct $\pi \pi$ transitions between the charmonium triplet $1 D$ and $1 S$ states. The BES result implies ${ }^{56}$ $\mathcal{B}\left(\psi\left(1^{3} D_{2}\right) \rightarrow \pi^{+} \pi^{-} J / \psi\right) \sim(20-40) \%$ which would make it easier to accommodate BELLE's results in the $1^{3} D_{2}$ interpretation of the $X(3872)$. The observed dipion mass distribution does not fit the shape for $1 D_{2} \rightarrow 1 S$ transitions predicted by the multipole expansion model (see Fig. 15). However, some dynamical effects beyond this model can alter the dipion distribution. Finally, the $\psi\left(1^{3} D_{2}\right)$ interpretation is disfavored by the mass predictions from potential model. This is illustrated in Fig. 16. All potential models but one predict the $\psi\left(1^{3} D_{2}\right)$ mass to be about $70 \mathrm{MeV}$ lower then the $X(3872)$ mass. The model by Fulcher ${ }^{71}$ predicts this mass right at the observed value, however it overestimates the $\psi\left(1^{3} D_{1}\right)$ mass by similar amount. In other words, none of the existing calculations can accommodate the $X(3872)$ and $\psi(3770)$ as spin 2 and 1 members of the $1^{3} D_{J}$ triplet. Coupled channel effects and $1^{3} D_{1}-2^{3} S_{1}$ mixing can increased the mass splitting relatively to the naïve potential model calculations. Quantitative estimates of these effects are needed.

The mass of the $\chi_{c}\left(2^{3} P_{1^{++}}\right)$state is predicted by the potential models to be higher than the $X(3872)$ mass (see Fig. 16). Thus, significant coupled channel effects would need to be at work for this interpretation as well. Decays of $B^{ \pm}$to $K^{ \pm} \chi_{c}\left(1^{3} P_{1}\right)$ are observed with a rate comparable to $K^{ \pm} \psi(2 S)$ and $K^{ \pm} J / \psi(1 S) \cdot{ }^{20}$ Therefore, decays to $K^{ \pm} \chi_{c}\left(2^{3} P_{1}\right)$ should also occur. If the $X(3872)$ is the $\chi_{c}\left(2^{3} P_{1}\right)$ state then the ratio $\mathcal{B}\left(X(3872) \rightarrow \gamma J / \psi\left(1^{3} S_{1}\right)\right) /$ $\mathcal{B}\left(X(3872) \rightarrow \pi^{+} \pi^{-} J / \psi\left(1^{3} S_{1}\right)\right)$ should be large. The photon transition here is of an E1 type and is observed with a $21 \%$ branching ratio in the $b \bar{b}$ system. Barnes and Godfrey predict $3.5 \%$ for the $\chi_{c}\left(2^{3} P_{1}\right)$ state. ${ }^{56}$ The $\chi_{c}\left(2^{3} P_{1}\right) \rightarrow \pi^{+} \pi^{-} J / \psi\left(1^{3} S_{1}\right)$ transition is of an isospin violating type and the branching ratio must be small even in the charmonium system. The BELLE experiment does not see evidence for significant $X(3872) \rightarrow \gamma J / \psi\left(1^{3} S_{1}\right)$ branching ratio. Quantitative results should be soon available. ${ }^{73}$

Finally, $X(3872)$ could be a $c \bar{c} g$ hybrid state, ${ }^{64,56}$ though the masses of the hybrid states are predicted to be significantly higher than the observed mass.

The molecular, conventional charmonium or hybrid charmonium interpretations of the $X(3872)$ should not be viewed as mutually exclusive options. For example, the production rate for molecular charmonium in $B$ decays is expected to be small. However, the production rate can be enhanced by mixing of the molecular system with conventional charmo- 
nium states ${ }^{74,64,65}$ (e.g. with $\chi_{c}\left(2^{3} P_{1}\right)$ for the $1^{++}$ molecule). Mixing would influence the pattern of decay branching ratios as well. ${ }^{74,64,56}$

More experimental studies of $X(3872)$ production and decays are needed to clarify nature of this state.

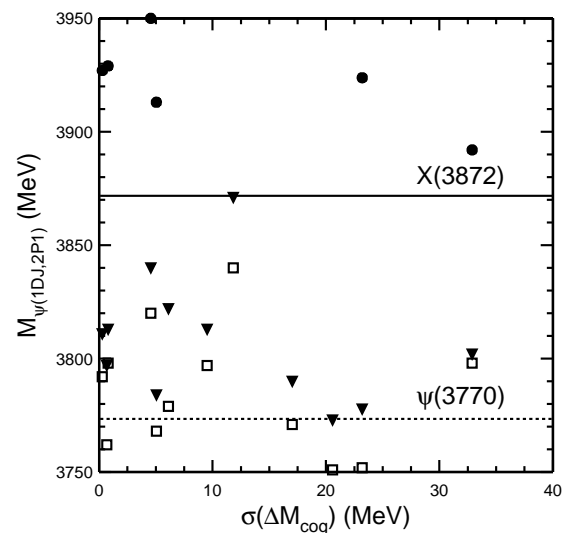

Figure 16. The predicted masses (vertical axis) of the $\psi\left(1^{3} D_{1}\right)$ (squares), $\psi\left(1^{3} D_{2}\right)$ (triangles) and $\chi_{c}\left(2^{3} P_{1}\right)$ (circles) states versus overall quality of the potential model expressed as RMS of the differences for the center-of-gravity masses of long-lived $c \bar{c}$ states (horizontal axis). The measured masses of $\psi(3770)$ and $X(3872)$ are indicated by horizontal bars. Predictions for the $1 \mathrm{~S}, 2 \mathrm{~S}$ and $1 \mathrm{P}$ masses are included in the RMS calculation for all models displayed here. ${ }^{72}$

\section{Summary and Outlook}

Heavy quarkonium physics has been recently experimentally revitalized. Large data samples collected in $e^{+} e^{-}$and $\bar{p} p$ annihilation by BES-II $(c \bar{c})$, CLEOIII $(b \bar{b})$ and E835 $(c \bar{c})$ are still being analyzed. The $B$-meson gateway to charmonium is now wide open with $3 \times 10^{8} B$ decays recorded by BELLE and BABAR. There has been similar progress in theory by the development of NRQCD and much improved calculations with QCD on the lattice.

More results for charmonium are expected in the future. In addition to more results from the BESII experiment, the CLEO-c program will contribute greatly. The first CLEO-c results have been presented. Run-II data from $\mathrm{CDF}$ are proving to be useful as well. In the farther future, $\mathrm{BTeV}$ and $\mathrm{LHCb}$ are likely to contribute to quarkonium physics. The BES-III/BEPC-II project was recently approved in China. There is also a proposal for a new dedicated $\bar{p} p$ machine to explore charmonium physics (PANDA at GSI).

Prospects for more $b \bar{b}$ data are not as well de- fined. In principle, CLEO at CESR could go back to the high energy running and accumulate more statistics for the narrow $\Upsilon$ resonances. BELLE and BABAR could accumulate $\Upsilon$ data with even higher rates if their $B$-factories are ever utilized to produce these states.

The discovery of the $X(3872)$ particle by BELLE, and its likely compound nature, underlines the importance of the heavy quarkonium systems. Previously charmonium played a crucial role in the solidification of the naïve quark model. It will not be a big surprise if it provides the first convincing proof for the existence of hadronic systems beyond the naïve quark model.

\section{Acknowledgments}

I would like to thank many colleagues from BABAR, BELLE, BES, CDF, CLEO, E835, H1 and ZEUS for providing me with information contained in this report. I apologize for not being able to discuss all the recent experimental results because of the length limitations. I would also like to thank Steve Godfrey, Jonathan Rosner, Mikhail Voloshin and Tung-Mow Yan for discussions concerning theoretical aspects. Special thanks to Sheldon Stone for help in refining this manuscript.

\section{References}

1. Y. Grossman, "Beyond Standard Model sensitivity in K and B Physics", talk presented at Lepton Photon 2003.

2. P. Lepage, "Lattice Gauge Theory", talk presented at Lepton Photon 2003.

3. W. E. Caswell, G. P. Lepage, Phys. Lett. B167, 437 (1986); B. A. Thacker, G. P. Lepage, Phys. Rev. D43, 196 (1991); G. T. Bodwin, E. Braaten, G. P. Lepage, Phys. Rev. D51, 1125 (1995); erratum D55, 5853 (1997); N. Brambilla, A. Pineda, J. Soto, A. Vairo, Nucl. Phys. B566, 275 (2000).

4. For a recent review see: M. Krämer, Prog. Part. Nucl. Phys. 47, 141 (2001).

5. E. L. Berger, D. Jones, Phys. Rev. D23, 1521 (1981); R. Baier, R. Rückl, Phys. Lett. B102, 364 (1981).

6. H. Fritzsch, Phys. Lett. B67, 217 (1977); F. Halzen, Phys. Lett. B69, 105 (1977); M. Glück, J. F. Owens, E. Reya, Phys. Rev. D17, 2324 (1978); G. A. Schuler, R. Vogt, Phys. Lett. B387, 181 (1996); J. F. Amundson, O. J. Eboli, E. M. Gregores, F. Halzen, Phys. Lett. B390, 323 (1997).

7. C. Adloff et al. (H1), papers contributed to LeptonPhoton 2003: "Diffractive Photoproduction of $\psi(2 \mathrm{~S})$ 
Mesons at HERA", $\sharp 151$; "Inelastic Leptoproduction of $\mathrm{J} / \psi$ Mesons at HERA", $\sharp 156$; "Inelastic Photoproduction of $\mathrm{J} / \psi$ Mesons at HERA", $\sharp 157$; "Diffractive Photoproduction of $\mathrm{J} / \psi$ Mesons with Large Momentum Transfer at HERA", $\sharp 162$; "Elastic Photoproduction of $\mathrm{J} / \psi$ Mesons", $\sharp 188$; S. Chekanov et al. (ZEUS), papers contributed to Lepton-Photon 2003: "Measurements of inelastic $\mathrm{J} / \psi$ and $\psi^{\prime}$ photoproduction at HERA", $\sharp 91$; "Measurement of proton-dissociative diffractive photoproduction of $\mathrm{J} / \psi$ mesons at HERA", $\sharp 106$; "Measurement of inelastic $\mathrm{J} / \psi$ production in deep inelastic scattering at HERA", $\sharp 109$; "Measurement of inelastic J/ $\psi$ helicity distribution at HERA", $\sharp 111$.

8. C. Baglin et al. (R704), Phys. Lett. B172, 455 (1986).

9. T. A. Armstrong et al. (E760), Nucl. Phys. B373, 35 (1992).

10. S. Bagnasco et al. (E835), Phys. Lett. B533, 237 (2002); N. Pastrone for the E835 at XXXVIIIth Recontres de Moriond, Les Arcs, Savoie, France, March 22-29, 2003, hep-ex/0306032.

11. M. Ambrogiani et al. (E835), Phys. Rev. D65, 052002 (2002); C. Patrignani at International Workshop on Heavy Quarkonium at Fermilab, Batavia, IL, USA, September 20-22, 2003.

12. M. Andreotti et al. (E835), Phys. Rev. Lett. 91, 091801 (2003).

13. M. Ambrogiani et al. (E835), Phys. Lett. B566, 45 (2003).

14. M. Ambrogiani et al. (E835), Phys. Rev. D64, 052003 (2001).

15. C. Edwards et al. (Crystal Ball), Phys. Rev. Lett. 48, 70 (1982).

16. C. Baglin et al. (R704), Phys. Lett. B171, 135 (1986).

17. T. A. Armstrong et al. (E760), Phys. Rev. Lett. 69, 2337 (1992).

18. See e.g. A. Martin, J.-M. Richard CERN Cour. 43N3, 17 (2003).

19. Rosanna Cester, private communication.

20. K. Hagiwara et al. (PDG), Phys. Rev. D66, 010001 (2002).

21. J. Z. Bai et al. (BES), Phys. Lett. B555, 174 (2003).

22. C. Wagner for BABAR at XXXVIIIth Recontres de Moriond, Les Arcs, Savoie, France, March 22-29, 2003, hep-ex/0305083; E. Robutti for BABAR at International Europhysics Conference on High Energy Physics EPS, Aachen, Germany, July 17-23, 2003.

23. F. Fang et al. (BELLE), Phys. Rev. Lett. 90, 071801 (2003), hep-ex/0208047.

24. The preliminary CLEO-c result for the $\eta_{c}$ mass, $(2976.1 \pm 2.4 \pm 3.3) \mathrm{MeV}$, comes from the photon energy measurement in $\psi^{\prime} \rightarrow \gamma \eta_{c}$ (see the text).

25. S. K. Choi et al. (BELLE), Phys. Rev. Lett. 89, 102001 (2002), Erratum-ibid. 89, 129901 (2002).

26. K. Abe et al. (BELLE), Phys. Rev. Lett. 89, 142001 (2002); K. Abe et al., BELLE-PREPRINT-2003-7,
KEK-PREPRINT-2003-24, hep-ex/0306015.

27. K. Abe et al. (BELLE), paper contributed to LeptonPhoton 2003, "A study of double $c \bar{c}$ production in $e^{+} e^{-}$annihilation at $\sqrt{s} \approx 10.6 \mathrm{GeV} ", \sharp 274$;

T. Uglov and R. Seuster for BELLE collaboration, talks presented at International Europhysics Conference on High Energy Physics EPS, Aachen, Germany, July 17-23, 2003.

28. E. Braaten, J. Lee, Phys. Rev. D67, 054007 (2003); K.-Y. Liu, Z.-G. He, K.-T. Chao, Phys. Lett. B557, 45 (2003); G. T. Bodwin, J. Lee, E. Braaten, Phys. Rev. D67, 054023 (2003); Phys. Rev. Lett. 90, 162001 (2003); A. B. Kaidalov, JETP Lett. 77, 349 (2003), Pisma Zh. Eksp. Teor. Fiz. 77, 417 (2003), hep-ph/0301246; A. V. Berezhnoy, A. K. Likhoded, hep-ph/0303145; K.-Y. Liu, Z.-G. He, K.-T. Chao, Phys. Rev. D68, 031501 (2003); K. Hagiwara, E. Kou, C.-F. Qiao, Phys. Lett. B570, 39 (2003); A. V. Luchinsky, hep-ph/0305253; S. J. Brodsky, A. S. Goldhaber, J. Lee, Phys. Rev. Lett. 91, 112001 (2003); B. L. Ioffe, D. E. Kharzeev, hep-ph/0306062; S. Fleming, A. K. Leibovich, T. Mehen CMU-HEP-03-06, FERMILAB-PUB-03069-T, hep-ph/0306139; C.-F. Qiao, J.-X. Wang, hep-ph/0308244.

29. J. Ernst et al. (CLEO), CLEO-CONF-03-05, hepex/0306060, paper contributed to Lepton-Photon 2003, "Observation of $\eta_{c}{ }^{\prime}$ Production in Two-photon Fusion at CLEO", $\sharp 37$.

30. References are ordered according to the increasing value of $R_{H F}$ given in square brackets. H. Ito, Prog. of Theor. Phys. 84, 94 (1990) [0.29]; T. A. Lahde, Nucl. Phys. A714, 183 (2003) [0.37]; S. Godfrey, N.Isgur, Phys. Rev. D32, 189 (1985) [0.46]; S. N. Jena, Phys. Lett. B123, 445 (1983) [0.48]; J. Carlson, J. B. Kogut, V.R.Pandharipande, Phys. Rev. D28, 2807 (1983) [0.55]; T. A. Lahde, C. J. Nyfalt, D. O. Riska Nucl. Phys. A645, 587 (1999) [0.56]; S. N. Gupta, W. W. Repko, C. J. Suchyta III, Phys. Rev. D39, 974 (1989) [0.61]; D. Beavis, S.-Y. Chu, B. R. Desai, P. Kaus, Phys. Rev. D20, 2345 (1979) [0.62]; N. Barik, S. N. Jena, Phys. Rev. D24, 680 (1981) [0.70]; S. N. Gupta, S. F. Radford, W. W. Repko, Phys. Rev. D26, 3305 (1982), Phys. Rev. D30, 2424 (1984) [0.73]; L. P. Fulcher, Phys. Rev. D44, 2079 (1991) [0.74]; J. S. Kang, Phys. Rev. D20, 2978 (1979) [0.76]; J. Baacke, Y. Igarashi, G. Kasperidus Z. Phys. C13, 131 (1982) [0.80]; S. N. Gupta, S. F. Radford, W. W. Repko, Phys. Rev. D34, 201 (1986) [0.83]; D. Ebert, R. N. Faustov, and V. O. Galkin Phys. Rev. D67, 014027 (2003) [0.84].

31. A. M. Badalian, B. L. G. Bakker, Phys. Rev. D67, 071901 (2003).

32. S. Recksiegel, Y. Sumino, TU-691, TUM-HEP-50803, hep-ph/0305178.

33. M. Okamoto et al. (CP-PACS Collaboration) Phys. Rev. D65, 094508 (2002). 
34. A. Heister et al. (ALEPH), Phys. Lett. B530, 56 (2002); M. Chapkine et al. (DELPHI), paper contributed to Lepton-Photon 2003, "Search for $\eta_{b}$ in two-photon collisions with the DELPHI detector", $\sharp 62$.

35. The following sample of potential model predictions for the M1 matrix elements (ordered according to the publication date) is displayed in Fig. 8: V. Zambetakis, N.Byers, Phys. Rev. D28, 2908 (1983); H. Grotch, D. A. Owen, K. J. Sebastian, Phys. Rev., D30, 1924 (1984) (2 entries: scalar and vector confinement potential); S. Godfrey, N. Isgur, Phys. Rev. D32, 189 (1985) (2 entries: based on quoted transition moments and wave functions respectively); X. Zhang, K. J. Sebastian, H. Grotch, Phys. Rev. D44, 1606 (1991) (2 entries: scalar-vector and pure scalar confinement potential); D. Ebert, R. N. Faustov, V. O. Galkin Phys. Rev. D67, 014027 (2003); T. A. Lahde, Nucl. Phys. A714, 183(2003) (2 entries: without and with exchange currents).Values of the M1 matrix elements in the $b \bar{b}$ system are displayed for the photon energies and $b$ quark mass assumed in S. Godfrey, J. L. Rosner, Phys. Rev. D64, 074011 (2001), Erratum-ibid. D65, 039901 (2002).

36. The following sample of potential model predictions for the E1 matrix elements (ordered according to the publication date) is displayed in Fig. 9: D. Pignon, C. A. Piketty, Phys. Lett. 74B, 108 (1978); E. Eichten, K. Gottfried, T. Kinoshita, K. D. Lane, T. M. Yan, Phys. Rev. D21, 203 (1980); W. Buchmuller, G. Grunberg, S.-H. Tye Phys. Rev. Lett. 45, 103 (1980), Phys. Rev. D24, 132 (1981); C. Quigg, J. L. Rosner, Phys. Rev. D23, 2625 (1981) (2 entries: $c \bar{c}, b \bar{b}$ potential respectively); J. Baacke, Y. Igarashi, G. Kasperidus, Z. Phys. C13, 131 (1982); R. McClary, N. Byers, Phys. Rev. D28, 1692 (1983); P. Moxhay, J. L. Rosner, Phys. Rev. D28, 1132 (1983); H. Grotch, D. A. Owen, K. J. Sebastian, Phys. Rev. D30, 1924 (1984); S. N. Gupta, S. F. Radford, W. W. Repko, Phys. Rev. D26, 3305 (1982), Phys. Rev. D30, 2424 (1984); S. N. Gupta, S. F. Radford, W. W. Repko, Phys. Rev. D34, 201 (1986); M. Bander, D. Silverman, B. Klima, U. Maor, Phys. Lett. B134, 258 (1984), Phys. Rev. D29, 2038 (1984), Phys. Rev. D36, 3401 (1987); W. Kwong, J. L. Rosner, Phys. Rev. D38, 279 (1988); L. P. Fulcher, Phys. Rev. D37, 1259 (1988); S. N. Gupta, W. W. Repko, C. J. Suchyta III, Phys. Rev. D39, 974 (1989); L. P. Fulcher, Phys. Rev. D42, 2337 (1990); A. K. Grant, J. L. Rosener, E. Rynes, Phys. Rev. D47, 1981 (1993); T. A. Lahde, Nucl. Phys. A714, 183(2003).

37. K. Gottfried, Phys. Rev. Lett. 40, 598 (1978); and Yan. $^{38}$

38. T. M. Yan, Phys. Rev. D22, 1652 (1980).

39. E. Eichten, K. Gottfried, Phys. Lett. B66, 286 (1977).

40. H. Severini et al. (CLEO), CLEO-CONF-03-06, con- tributed to Lepton Photon 2003, hep-ex/0307034

41. M. B. Voloshin, Mod. Phys. Lett. A18, 1067 (2003).

42. T. M. Yan, Y. P. Kuang, Phys. Rev. D24, 2874 (1981).

43. M. B. Voloshin Nucl. Phys. B154, 365 (1979); M. B. Voloshin, V. Zakharov Phys. Rev. Lett. 45, 688 (1980); V. A. Novikov, M. A. Shifman, Z. Phys. 8, 43 (1981); M. B. Voloshin Pisma Zh. Eksp. Teor. Fiz. 37, 58 (1983) [JETP Lett. 37, 69 (1983)].

44. S. E. Csorna et al., CLEO-CONF-02-06, Contributed to 31st International Conference on High Energy Physics (ICHEP 2002), Amsterdam, The Netherlands, 24-31 Jul 2002, hep-ex/0207060.

45. CLEO collaboration, private communication.

46. The following sample of potential models (ordered according to the increasing value of $\left.\sigma\left(\Delta M_{\operatorname{cog}}\right)\right)$ is displayed in Fig. 11: D. Ebert, R. N. Faustov, V. O. Galkin, Phys. Rev. D62, 034014 (2000); W. Kwong, J. L. Rosner, Phys. Rev. D38, 279 (1988); L. P.Fulcher, Phys. Rev. D42, 2337 (1990); P. Moxhay, J. L. Rosner, Phys. Rev. D28, 1132 (1983); L. P. Fulcher, Phys. Rev. D44, 2079 (1991); S. Godfrey, N. Isgur, Phys. Rev. D32, 189 (1985); E. Eichten, F. Feinberg, Phys. Rev. D23, 2724 (1981); T. A. Lahde, Nucl. Phys. A714, 183 (2003); J. R. Hiller, Phys. Rev. D30, 1520 (1984); W. Buchmuller, Phys. Lett. B112, 479 (1982); T. A. Lahde, C. J. Nyfalt, D. O. Riska, Nucl. Phys. A645, 587 (1999); J. S. Kang, Phys. Rev. D20, 2978 (1979); M. Hirano, Prog. of Theor. Phys. 83, 575 (1990); J. Baacke, Y. Igarashi, G. Kasperidus, Z. Phys. C13, 131 (1982).

47. S. Godfrey, J. L. Rosner Phys. Rev. D64, 097501 (2001), Erratum-ibid. D66, 059902 (2002).

48. J. L. Rosner, Phys. Rev. D67, 097504 (2003)

49. P. Moxhay, Phys. Rev. D37, 2557 (1988).

50. P. Ko, Phys. Rev. D47, 208 (1993).

51. M. B. Voloshin, Phys. Lett. B562, 68 (2003).

52. J. Z. Bai et al. (BES) hep-ex/0307028.

53. Y. P. Kuang, Phys. Rev. D65, 094024 (2002); Y. P. Kuang, T.M.Yan, Phys. Rev. D41, 155 (1990).

54. S. K. Choi et al. (BELLE), hep-ex/0309032; K. Abe et al. (BELLE), hep-ex/0308029, paper contributed to Lepton-Photon 2003, "Observation of a new narrow charmonium state in exclusive $B^{ \pm} \rightarrow$ $K^{ \pm} \pi^{+} \pi^{-} J / \psi$ decays", $\sharp 307$.

55. M. B. Voloshin, hep-ph/0309307.

56. T. Barnes, S. Godfrey, hep-ph/0311162.

57. G. Bauer (CDF), at International Workshop on Heavy Quarkonium at Fermilab, Batavia, IL, USA, September 20-22, 2003.

58. M. Bander, G. L. Shaw, P. Thomas, Phys. Rev. Lett. 36, 695 (1976); M. B. Voloshin, L. B. Okun JETP Lett. 23, 333 (1976), Pisma Zh. Eksp. Teor. Fiz. 23, 369 (1976); A. De Rujula, H. Georgi, S. L. Glashow, Phys. Rev. Lett. 38, 317 (1977).

59. E. Eichten, K. Gottfried, T. Kinoshita, K. D. Lane, Tung-Mow Yan, Phys. Rev. D21, 203 (1980). 
60. A. Bradley, D. Robson, Phys. Lett. B93, 69 (1980); Z. Phys. C6, 57 (1980).

61. R. L. Jaffe, Phys. Rev. D15, 267 (1977); J. Weinstein, N. Isgur, Phys. Rev. Lett. 48, 659 (1982), Phys. Rev. D27, 588 (1982), Phys. Rev. D41, 2236 (1990).

62. N. A. Tornqvist, Phys. Rev. Lett. 67, 556 (1991), Z. Phys. C61, 525 (1994), hep-ph/0308277.

63. A. V. Manohar, M. B. Wise, Nucl. Phys. B339, 17 (1993); T. E. O. Ericson, G. Karl, Phys. Lett. B309, 426 (1993).

64. F. E. Close, P. R. Page, hep-ex/0309253.

65. E. Braaten, M. Kusunoki, hep-ph/0311147.

66. The data come from Fig.3a in BELLE's paper. ${ }^{54}$ The sideband distribution was subtracted here. The binning and normalization were also changed.

67. K. Abe et al. (BELLE), hep-ex/0307061.

68. F. Yuan, C.-F. Qiao, K.-T. Chao, Phys. Rev. D56, 329 (1997).

69. E. J. Eichten, K. Lane, C. Quigg, Phys. Rev. Lett. 89, 162002 (2002).

70. E. J. Eichten, at International Workshop on Heavy Quarkonium at Fermilab, Batavia, IL, USA, September 20-22, 2003.

71. L. P. Fulcher, Phys. Rev. D44, 2079 (1991).

72. The following sample of potential models (ordered according to the increasing value of $\left.\sigma\left(\Delta M_{\operatorname{cog}}\right)\right)$ is displayed in Fig. 16: H. Ito, Prog. of Theor. Phys. 84, 94 (1990); D. Ebert, R. N. Faustov, V. O. Galkin, Phys. Rev. D67, 014027 (2003); E. Eichten, F. Feinberg, Phys. Rev. D23, 2724 (1981); S. Godfrey, N. Isgur, Phys. Rev. D32, 189 (1985); T. A. Lahde, Nucl. Phys. A714, 183 (2003); N. Barik, S. N. Jena, Phys. Rev. D24, 680 (1981); W. Buchmuller, Phys. Lett. B112, 479 (1982); L. P. Fulcher, Phys. Rev. D44, 2079 (1991); J. Baacke, Y. Igarashi, G.Kasperidus, Z. Phys. C13, 131 (1982); J. R. Hiller, Phys. Rev. D30, 1520 (1984); M. Hirano, Prog. of Theor. Phys. 83, 575 (1990); T. A. Lahde, C. J. Nyfalt, D. O. Riska, Nucl. Phys. A645, 587 (1999).

73. BELLE collaboration, private communication.

74. S. Pakvasa, M. Suzuki, hep-ph/0309294. 